\title{
Soft gluon resummation for gluon-induced Higgs Strahlung
}

\author{
Robert V. Harlander, ${ }^{a}$ Anna Kulesza, ${ }^{b}$ Vincent Theeuwes ${ }^{b}$ and Tom Zirke ${ }^{a}$ \\ ${ }^{a}$ Fachbereich C, Bergische Universität Wuppertal, \\ 42097 Wuppertal, Germany \\ ${ }^{b}$ Institute for Theoretical Physics, WWU Münster, \\ D-48149 Münster, Germany \\ E-mail: robert.harlander@uni-wuppertal.de, \\ anna.kulesza@uni-muenster.de, vthee_01@uni-muenster.de, \\ t.zirke@uni-wuppertal.de
}

ABSTRACT: We study the effect of soft gluon emission on the total cross section predictions for the $g g \rightarrow H Z$ associated Higgs production process at the LHC. To this end, we perform resummation of threshold corrections at the NLL accuracy in the absolute threshold production limit and in the threshold limit for production of a $Z H$ system with a given invariant mass. Analytical results and numerical predictions for various possible LHC collision energies are presented. The perturbative stability of the results is verified by including universal NNLL effects. We find that resummation significantly reduces the scale uncertainty of the $g g \rightarrow H Z$ contribution, which is the dominant source of perturbative uncertainty to $Z H$ production. We use our results to evaluate updated numbers for the total inclusive cross section of associated $p p \rightarrow Z H$ production at the LHC. The reduced scale uncertainty of the $g g \rightarrow H Z$ component translates into a decrease of the overall scale error by about a factor of two.

KeYwords: Higgs Physics, Resummation, QCD, Standard Model

ARXIV EPRINT: 1410.0217 


\section{Contents}

1 Introduction 1

$\begin{array}{lll}2 & \text { Formalism } & 3\end{array}$

3 Calculation of the resummed cross sections $\quad 7$

3.1 The invariant mass threshold limit: $Q$-approach $\quad 7$

3.2 The absolute threshold limit: $M$-approach 8

4 Results for the resummed cross section $\quad 10$

5 The total inclusive cross section for $Z H$ production 13

6 Conclusions $\quad 15$

\section{Introduction}

One of the main tasks for physics at the Run II of the LHC will be the precise determination of the properties of the newly found Higgs boson at about $125 \mathrm{GeV}[1,2]$. Apart from the enormous experimental activity behind the relevant measurements, this requires a detailed theoretical understanding of the underlying processes. Tremendous efforts have already been devoted in this direction, the most central of which are collected in the three reports of the LHC Higgs Cross Section Working Group [3-5].

Associated production of a Higgs boson with a weak gauge boson (Higgs Strahlung for short) may in fact turn out a key channel for studying Higgs properties. The decay products of the final state gauge boson allow for the separation of this process from the other production channels, possibly with an additional restriction to the kinematical regime of boosted Higgs bosons [6]. An interesting feature of Higgs Strahlung is that there are actually two variants of it, namely $Z H$ and $W H$ production. While the next-to-leading order (NLO) QCD corrections [7-12] for these two processes are identical, at next-to-NLO (NNLO) [13-16] the $Z H$ process receives corrections from gluon-initiated, quark-loop mediated contributions, which are absent for $W H$ production, see figure 1 . They are the main subject of this paper and will be referred to as $g g \rightarrow H Z$ in what follows. ${ }^{1}$ In the Standard Model (SM), these corrections are completely dominated by top- and bottom-quark loops and can amount to about $8 \%$ in the total cross section. In extended theories, this contribution can differ significantly from the SM prediction (see, e.g., refs. [17, 18]). One could therefore consider the ratio $\sigma_{W H} / \sigma_{Z H}$ as a probe for new physics, for example [18].

\footnotetext{
${ }^{1}$ Note that at higher orders, other partonic initial states have to be taken into account as well in order to cancel initial state singularities. However, as usual, we will still refer to them as $g g \rightarrow H Z$ in this paper, unless indicated otherwise.
} 

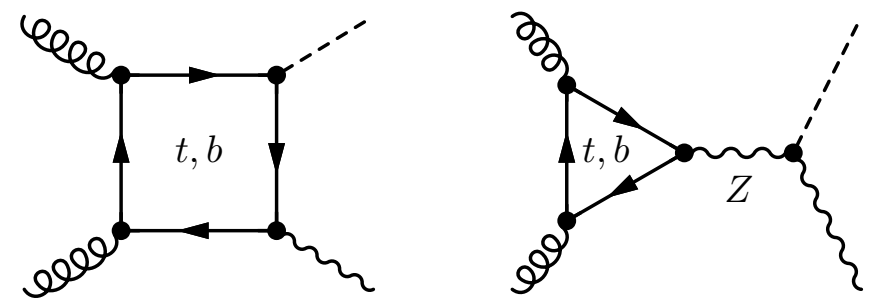

Figure 1. Sample Feynman diagrams for the subprocess $g g \rightarrow H Z$ to associated $Z H$ production.

The $g g \rightarrow H Z$ contribution induces a significant uncertainty to the $Z H$ cross section (and therefore also to the $W H / Z H$ ratio), since their renormalization and factorization scale dependence is not compensated by any lower order terms.

The NLO QCD corrections to this contribution (i.e. $\left.\mathcal{O}\left(\alpha_{\mathrm{s}}^{3}\right)\right)$ have been evaluated in ref. [19], albeit in the heavy-top limit, i.e., by letting $m_{t} \rightarrow \infty$ and $m_{b} \rightarrow 0$. They increase the gluon-initiated contribution by roughly $100 \%$; the NLO result still suffers from a scale uncertainty of $20-30 \%$, which corresponds to about $2-3 \%$ relative to the total $Z H$ cross section.

The gluon-induced $Z H$ process bears a striking similarity in the production mechanism to the well-known gluon fusion process $g g \rightarrow H$ (see, e.g. ref. [20]), also manifested in the numerical behaviour of the two processes. The substantial size of the NLO corrections ( $K$-factors of two) can be attributed to the soft gluon emission off the initial state, leading to logarithmic contributions which get large in the threshold limit for the production. By performing resummation of these corrections, one can not only take into account these logarithmic contributions to all orders in perturbation theory, but also reduce the theoretical uncertainty due to scale variation. It is the purpose of this paper to perform this resummation at the next-to-leading logarithmic (NLL) accuracy and match the resummed result to the NLO predictions, obtaining in this way the NLO+NLL result. We will also discuss the effect of the next-to-NLL (NNLL) corrections on the theoretical predictions.

Threshold resummation techniques have been used extensively in the recent years to improve the knowledge of Higgs production cross sections. The most prominent example is the inclusive Higgs production via gluon fusion, $g g \rightarrow H$ [21-32]. Resummation of threshold logarithms has also been used to improve the predictions for the $g g \rightarrow H$ process when the Higgs is produced at high transverse momentum and the accompanying jet remains unobserved [33-35]. For the process of interest here, i.e. the associated Higgs production with a weak gauge boson, threshold resummation has been performed so far only for the $q \bar{q}$-initiated partonic processes [36]. For an extensive discussion of the subject we refer the reader to refs. [3-5] and references therein.

In contrast to the process $g g \rightarrow H$ where the threshold is uniquely defined at the partonic center-of-mass energy $\hat{s}=M_{H}^{2}$, one may argue along two different lines in $Z H$ production. On the one hand, the absolute threshold for this process is given by the minimum of the invariant mass of the $Z H$ system, $\hat{s}_{\text {min }}=\left(M_{H}+M_{Z}\right)^{2}$. Indeed, the total cross section receives a large portion from the kinematical region around this threshold, 
partly due to the usual logarithmic enhancement from soft and collinear gluon radiation, but also due to the gluon luminosity which drops quite steeply towards larger values of the partonic center-of-mass energy. On the other hand, one may consider the production of the $Z H$ system at a definite value of the squared invariant mass $Q^{2} \equiv\left(p_{H}+p_{Z}\right)^{2}$, and resum the threshold logarithms with respect to that scale. From a formal point of view, this latter approach is in closer relation to the one for the Drell-Yan process considered at a fixed invariant mass of the lepton pair, or the $g g \rightarrow H$ process at a given Higgs mass.

We are going to present the formalism and the results for both of these approaches and discuss the differences between them. The behavior of the final results will allow us to define a preferred approach, in the sense that it allows for a more appropriate scale choice and therefore appears to be perturbatively better behaved. Both approaches lead to compatible results, however, which provides us with an important consistency check.

The remainder of the paper is structured as follows: in the next section we discuss the threshold resummation formalism, whereas in section 3 we present process-specific results needed to perform resummation at NLL. Section 4 is dedicated to the discussion of the numerical NLO+NLL predictions at the LHC. It also briefly addresses the universal part of the NNLL effects. Finally, in section 5 we update the total inclusive cross section predictions of associated $Z H$ production at the LHC; we conclude in section 6 .

\section{Formalism}

Let us consider the hadronic cross section $\Sigma$ for the production of a color-neutral system which involves a scale $W$, defined as a characteristic scale for the production threshold, cf. refs. [37-39]. In the following we will consider a $2 \rightarrow 2$ process in a pair-invariant kinematics, whenever appropriate. With the help of the hadronic and partonic threshold variables

$$
\tau \equiv W^{2} / s, \quad \hat{\tau} \equiv W^{2} / \hat{s}
$$

where $s$ and $\hat{s}$ are the hadronic and the partonic center-of-mass energy, respectively, this cross section can be written as

$$
\Sigma\left(W^{2}, \tau\right)=\sum_{i, j} \int \mathrm{d} x_{1} \mathrm{~d} x_{2} d \hat{\tau} \delta\left(\hat{\tau}-\frac{\tau}{x_{1} x_{2}}\right) f_{i}\left(x_{1}, \mu\right) f_{j}\left(x_{2}, \mu\right) \hat{\Sigma}_{i j}\left(W^{2}, \hat{\tau}, \mu^{2}\right),
$$

where we have set the renormalization and factorization scales equal to $\mu$ in order to simplify the discussion. The dependence on all relevant mass parameters, other than that related to the threshold scale, is implicit in eq. (2.2) and throughout the rest of this paper.

At higher orders in perturbation theory, the partonic cross section $\hat{\Sigma}_{i j}$ contains logarithmic terms originating from soft gluon emission that diverge in the threshold limit, i.e. for $\hat{\tau} \rightarrow 1$. Such terms clearly spoil the perturbative behavior of a naïve expansion in $\alpha_{\mathrm{s}}$ in the threshold region. However, these logarithmic terms can be treated systematically with the help of threshold resummation which is most conveniently formulated in Mellin space, 
where the hadronic cross section reads

$$
\begin{aligned}
\tilde{\Sigma}\left(W^{2}, N\right) & \equiv \int_{0}^{1} \mathrm{~d} \tau \tau^{N-1} \Sigma_{i j}\left(W^{2}, \tau\right) \\
& =\sum_{i, j} \tilde{f}_{i}\left(N+1, \mu^{2}\right) \tilde{f}_{j}\left(N+1, \mu^{2}\right) \tilde{\hat{\Sigma}}_{i j}\left(W^{2}, N, \mu^{2}\right) .
\end{aligned}
$$

The Mellin transform of a function $h(\ldots, x, \ldots)$ w.r.t. the variable $x$ is defined as usual as

$$
\tilde{h}(\ldots, N, \ldots) \equiv \int_{0}^{1} d x x^{N-1} h(\ldots, x, \ldots) .
$$

The moments of the hadronic (partonic) cross section are taken w.r.t. the variable $\tau(\hat{\tau})$, those of the parton distributions $f_{i}\left(x, \mu^{2}\right)$ are taken w.r.t. the partonic momentum fraction $x$. The Mellin transformation turns the terms that are logarithmic in $1-\tau$ (respectively $1-\hat{\tau}$ ) into logarithms of the Mellin variable $N$; it is the latter which are then resummed to all orders in $\alpha_{\mathrm{s}}$.

As the subject of this paper is the gluon-initiated component of associated $Z H$ production, $\sigma(g g \rightarrow H Z)$, it will be convenient to specialize the presentation to gluon-initiated processes in the following. The resummed partonic cross section then has the form:

$$
\begin{aligned}
& \tilde{\hat{\Sigma}}^{(\mathrm{res})}\left(W^{2}, N, \mu^{2}\right)=\tilde{\hat{\Sigma}}^{\mathrm{LO}}\left(W^{2}, N, \mu^{2}\right) \mathcal{C}_{W}\left(W^{2}, \mu^{2}\right) \\
& \times \Delta_{g}\left(W^{2}, N+1, \mu^{2}\right) \Delta_{g}\left(W^{2}, N+1, \mu^{2}\right) .
\end{aligned}
$$

The LO partonic cross section, $\hat{\Sigma}^{\mathrm{LO}}$, is obtained from Feynman diagrams with closed topand bottom-quark loops, see figure $1 .^{2}$ The Sudakov factor $\Delta_{g}$ describes the soft emission off an incoming gluon $g$; in the $\overline{\mathrm{MS}}$ factorization scheme it is given by, up to the NNLL level,

$$
\log \Delta_{g}\left(W^{2}, N, \mu^{2}\right)=\int_{0}^{1} d z \frac{z^{N-1}-1}{1-z} \int_{\mu^{2}}^{W^{2}(1-z)^{2}} \frac{d q^{2}}{q^{2}} A_{g}\left(\alpha_{\mathrm{S}}\left(q^{2}\right)\right)+D_{g}\left(\alpha_{\mathrm{S}}\left((1-z)^{2} W^{2}\right)\right)
$$

where the resummation coefficients have the following perturbative expansions:

$$
A_{g}\left(\alpha_{\mathrm{s}}\right)=\sum_{n \geq 1}\left(\frac{\alpha_{\mathrm{s}}}{\pi}\right)^{n} A_{g}^{(n)}, \quad D_{g}\left(\alpha_{\mathrm{s}}\right)=\sum_{n \geq 2}\left(\frac{\alpha_{\mathrm{s}}}{\pi}\right)^{n} D_{g}^{(n)} .
$$

Through NLL, only $A_{g}$ contributes, with the leading logarithmic (LL) and NLL coefficients given by $[45,46]\left(C_{A}=3, T_{R}=1 / 2\right)$

$$
A_{g}^{(1)}=C_{A}, \quad A_{g}^{(2)}=C_{A}^{2}\left(\frac{67}{36}-\frac{\pi^{2}}{12}\right)-\frac{5}{9} C_{A} T_{R} .
$$

\footnotetext{
${ }^{2}$ Note that the bottom-loop contributions could lead to a breaking of factorizaton at $1-\hat{\tau} \sim m_{b}^{2} / W^{2}$ which would be in conflict with eq. (2.5). However, in ref. [19] it was shown that these diagrams are absent for $m_{b}=0$ in Landau gauge. The genuine bottom-loop effects are thus suppressed by the bottom Yukawa coupling or by powers of $m_{b} / M$. Using vh@nnlo [40], we checked that their numerical effect is at the percent level, so that this issue can safely be ignored for the scope of this paper. We note in passing that also for the gluon fusion process, $g g \rightarrow H$, soft gluon resummation for the bottom-loop induced terms has been ignored so far. For a related discussion in the context of small- $p_{T}$ resummation, see refs. [41-44].
} 
At NNLL accuracy one requires the knowledge of $D_{g}^{(2)}$ and $A_{g}^{(n)}$ through $n=3$. Explicit results for these coefficients can be found in ref. [47-49]. In numerical applications, we use expansions of eq. (2.6) up to NLL, respectively NNLL terms in $N$, see eqs. (24) and (27) of ref. [21].

The final ingredient in eq. (2.5) is the process dependent perturbative function $\mathcal{C}_{W}$, which contains the "hard" contributions, i.e. terms that are constant in the large- $N$ limit. At NLL, they originate from NLO virtual and the non-logarithmic soft corrections. Correspondingly, $\mathcal{C}_{W}$ requires the knowledge of the threshold limit of the NLO cross section. It is well known that the latter can be cast into the form

$$
\hat{\Sigma}^{\mathrm{NLO}}=\int_{3}\left[\left.\mathrm{~d} \hat{\Sigma}^{\mathrm{R}}\right|_{\epsilon=0}-\left.\mathrm{d} \hat{\Sigma}^{\mathrm{A}}\right|_{\epsilon=0}\right]+\int_{2}\left[\mathrm{~d} \hat{\Sigma}^{\mathrm{V}}+\int_{1} \mathrm{~d} \hat{\Sigma}^{\mathrm{A}}\right]_{\epsilon=0}+\hat{\Sigma}^{\mathrm{C}}
$$

using the standard notation of $\mathrm{d} \hat{\Sigma}^{\mathrm{R}}$ for the real part of the corrections, $\mathrm{d} \hat{\Sigma}^{\mathrm{V}}$ for the virtual part, $\mathrm{d} \hat{\Sigma}^{\mathrm{A}}$ for the auxiliary singular dipole terms [50] and $\hat{\Sigma}^{\mathrm{C}}$ for the collinear counter terms. By construction of the dipole terms, the first integral on the r.h.s. vanishes faster in the soft limit than the remaining terms and therefore can be ignored. We have also confirmed this numerically using the specific matrix elements and subtraction terms. The remaining terms can be written as

$$
\hat{\Sigma}^{\mathrm{NLO}}(\hat{\tau})=\hat{\Sigma}_{\mathrm{reg}}^{V}(\hat{\tau})+2 \int_{\hat{\tau}}^{1} \mathrm{~d} x\left[\boldsymbol{P}^{g g}+\boldsymbol{K}^{g g}\right](x) \hat{\Sigma}^{\mathrm{LO}}(\hat{\tau} / x)+\ldots
$$

where the ellipse denotes terms that are sub-leading in the soft limit, and [50]

$$
\begin{aligned}
{\left[\boldsymbol{P}^{g g}+\right.} & \left.\boldsymbol{K}^{g g}\right](x) \\
= & \frac{\alpha_{\mathrm{s}}}{2 \pi}\left(2 C _ { A } \left\{2\left(\frac{\log (1-x)}{1-x}\right)_{+}-\left(\frac{1}{1-x}\right)_{+} \log \left(\frac{\mu^{2}}{\hat{s}}\right)\right.\right. \\
& \left.-\left[\frac{1-x}{x}-1+x(1-x)\right] \log \left(\frac{\mu^{2}}{(1-x)^{2} \hat{s}}\right)-\delta(1-x) \frac{\pi^{2}}{6}\right\} \\
& \left.-\delta(1-x)\left\{\left(\frac{11}{6} C_{A}-\frac{2}{3} T_{R} n_{l}\right) \log \left(\frac{\mu^{2}}{\hat{s}}\right)+\left(\frac{50}{9}-\frac{2 \pi^{2}}{3}\right) C_{A}-\frac{16}{9} T_{R} n_{l}\right\}\right)
\end{aligned}
$$

denotes process independent terms, while $\hat{\Sigma}_{\text {reg }}^{V}$ are the IR-subtracted virtual corrections to the total NLO cross section according to the method of dipole subtraction [50]. For loop-induced processes they typically require a genuine two-loop calculation.

Note that in Mellin space, the second term on the r.h.s. of eq. (2.10) factorizes into the Mellin transforms of $\hat{\Sigma}^{\mathrm{LO}}(x)$ and $x\left[\boldsymbol{P}^{g g}+\boldsymbol{K}^{g g}\right](x)$. The latter is easily evaluated using well-known relations for Mellin transforms (see, e.g., ref. [51]), and in the large/ $N$ limit consists of terms that are logarithmic or constant in $N$. For the terms constant in $N$ (as 
$N \rightarrow \infty)$ which is all that is needed here, we find

$$
\begin{aligned}
U\left(W^{2}\right) \equiv 2 \int \mathrm{d} x x^{N-1} x & {\left.\left[\boldsymbol{P}^{g g}+\boldsymbol{K}^{g g}\right](x)\right|_{N \text {-const }} } \\
=\frac{\alpha_{\mathrm{s}}}{\pi}\left\{\left[\frac{2}{3} T_{R} n_{l}-\right.\right. & \left.\left(\frac{11}{6}-2 \gamma_{E}\right) C_{A}\right] \log \left(\frac{\mu^{2}}{W^{2}}\right) \\
& \left.-\left(\frac{50}{9}-\frac{2 \pi^{2}}{3}-2 \gamma_{E}^{2}\right) C_{A}+\frac{16}{9} T_{R} n_{l}\right\},
\end{aligned}
$$

where, as usual, $\gamma_{E}=0.5772157 \ldots$ denotes Euler's constant. Eq. (2.12) is in agreement with the expression for the $\tilde{I}_{g}^{(1)}$ operator introduced in ref. [52] after accounting for terms originating from the Mellin transform and adding universal contributions containing $1 / \epsilon$ poles in the dipole subtraction method.

The validity of the threshold resummed cross section $\hat{\Sigma}^{(\text {res })}$ is restricted to the soft region $\hat{\tau} \approx 1$. In order to arrive at a result which is valid for general $\hat{\tau}$, one should combine the resummed and the fixed-order expression without double counting terms that are contained in both. This is achieved by subtracting the perturbative expansion of the resummed cross section. The resummation-improved hadronic cross section therefore reads:

$$
\begin{aligned}
\Sigma^{(\text {f.o.+l.a. })}\left(W^{2}, \tau\right)= & \Sigma^{\text {(f.o.) }}\left(W^{2}, \tau\right)+\sum_{i j} \int_{\mathrm{CT}} \mathrm{d} N \tau^{-N} \tilde{f}_{i}\left(N+1, \mu^{2}\right) \tilde{f}_{j}\left(N+1, \mu^{2}\right) \\
& \times\left[\tilde{\hat{\Sigma}}_{i j}^{(\text {l.a. })}\left(N, \mu^{2}\right)-\left.\tilde{\hat{\Sigma}}_{i j}^{(\text {l.a. })}\left(N, \mu^{2}\right)\right|_{\text {(f.o.) }}\right]
\end{aligned}
$$

where "(f.o.)" denotes the fixed order result, while "(l.a.)" marks the resummed expression. The inverse Mellin transform can be evaluated numerically using a contour CT in the complex- $N$ space according to the "Minimal Prescription" method developed in ref. [53].

As outlined in the introduction of this paper, we will pursue two alternative approaches for the resummation of threshold logarithms in the $g g \rightarrow H Z$ process which essentially differ by the specific choice for $W$.

In the first approach, dubbed $Q$-approach in the following, we will consider threshold resummation for the production of the $Z H$ system at a fixed invariant mass $Q$, i.e., we are going to set

$$
\begin{aligned}
W & =Q \equiv \sqrt{\left(p_{H}+p_{Z}\right)^{2}}, \quad \tau=\tau_{Q} \equiv Q^{2} / s, \\
\Sigma\left(W^{2}, \tau\right) & =\frac{\mathrm{d} \sigma_{g g \rightarrow H Z}}{\mathrm{~d} Q^{2}},
\end{aligned}
$$

and analogously for the partonic quantities. $\sigma_{g g \rightarrow H Z}$ is the cross section for the process $g g \rightarrow H Z$, and $p_{Z}$ and $p_{H}$ are the final-state four-momenta. The logarithmic terms which are treated by the resummation formalism are of the form

$$
\alpha_{\mathrm{s}}^{2+n}\left(\frac{\log ^{m}\left(1-\hat{\tau}_{Q}\right)}{1-\hat{\tau}_{Q}}\right)_{+}, \quad m \leq 2 n-1
$$

in this case. The +-distribution is defined in the usual way as

$$
\int_{0}^{1} \mathrm{~d} \hat{\tau}_{Q} g\left(\hat{\tau}_{Q}\right)\left[f\left(\hat{\tau}_{Q}\right)\right]_{+} \equiv \int_{0}^{1} \mathrm{~d} \hat{\tau}_{Q}\left[g\left(\hat{\tau}_{Q}\right)-g(1)\right] f\left(\hat{\tau}_{Q}\right) .
$$

The total cross section can be obtained upon subsequent integration over $Q^{2}$. 
In the second approach, we will consider the ( $Q^{2}$-integrated) total inclusive cross section for the process $g g \rightarrow H Z$, and resum the logarithms relative to the absolute threshold for the production of the $Z H$ pair, $M^{2}=\left(M_{H}+M_{Z}\right)^{2}$. Correspondingly, we will refer to this procedure as $M$-approach and set

$$
\begin{aligned}
W & =M \equiv M_{H}+M_{Z}, \quad \tau=\tau_{M} \equiv M^{2} / s, \\
\Sigma\left(W^{2}, \tau\right) & =\sigma_{g g \rightarrow H Z} .
\end{aligned}
$$

In this case, the divergences at threshold occur in the form

$$
\alpha_{\mathrm{s}}^{2+n} \log ^{m}\left(1-\hat{\tau}_{M}\right), \quad m \leq 2 n .
$$

The difference between the two approaches has been studied in the literature in the context of $t \bar{t}$ production $[39,54,55]$. In particular, in ref. [39] it has been shown that the total cross section obtained by integrating the $Q$-approach result over the invariant mass contains the same leading logarithmic terms as those in the $M$-approach, but differs by power-suppressed terms. In what follows, we pursue the calculation in both approaches and treat them in a complimentary way. In practice, however, since the $Q$-approach allows for a $Q$-dependent choice of the renormalization and factorization scale, it appears to be better suited to describe the physics of the process at hand.

\section{Calculation of the resummed cross sections}

Let us now discuss the various specific ingredients that enter the expressions for the threshold-resummed cross sections at NLL accuracy in the two approaches.

\subsection{The invariant mass threshold limit: $Q$-approach}

At LO, the partonic invariant mass distribution is proportional to a $\delta$-function,

$$
\frac{\mathrm{d} \hat{\sigma}_{g g \rightarrow H Z}^{\mathrm{LO}}}{\mathrm{d} Q^{2}}=\hat{\sigma}_{g g \rightarrow H Z}^{\mathrm{LO}}\left(\hat{s}, M_{H}, M_{Z}\right) \delta\left(\hat{s}-Q^{2}\right),
$$

where $\hat{\sigma}_{g g \rightarrow H Z}^{\mathrm{LO}}$ is the total inclusive cross section for $Z H$ production at LO [13, 56-58]. The Mellin transform is therefore constant in $N$ :

$$
\frac{\mathrm{d} \tilde{\hat{\sigma}}_{g g \rightarrow H Z}^{\mathrm{LO}}}{\mathrm{d} Q^{2}}=\frac{\hat{\sigma}_{g g \rightarrow H Z}^{\mathrm{LO}}\left(\hat{s}=Q^{2}, M_{H}, M_{Z}\right)}{Q^{2}} .
$$

Note that due to the form of the LO cross section in eq. (3.1), the convolution in eq. (2.10) turns into a simple product proportional to the LO cross section $\hat{\sigma}_{g g \rightarrow H Z}^{\mathrm{LO}}\left(Q^{2}, M_{H}, M_{Z}\right)$ and the term $\left[\boldsymbol{P}^{g g}+\boldsymbol{K}^{g g}\right](x)$ taken at $x=\hat{\tau}_{Q}$. The exact partonic LO cross section $\hat{\sigma}_{g g \rightarrow H Z}^{\mathrm{LO}}$ is implemented in vh@nnlo $[18,40]$ in terms of Passarino-Veltman functions which are evaluated with the help of LoopTools [59]. We use the corresponding subroutines of vh@nnlo for our numerical analysis. 
Obviously, the kinematics of the virtual corrections is the same as for the LO cross section, and we can write

$$
\hat{\tilde{\Sigma}}_{\mathrm{reg}}^{V}\left(Q^{2}, N\right)=\frac{\hat{\sigma}_{\text {virt }}}{Q^{2}}
$$

where the virtual cross section at NLO has been evaluated in the limit of an infinitely heavy top quark [19]:

$$
\begin{gathered}
\hat{\sigma}_{\text {virt }}=K(\hat{s}) \cdot \hat{\sigma}_{g g \rightarrow H Z}^{\mathrm{LO}}+\hat{\sigma}_{(\text {virt,red }),} \\
\text { with } \quad K(\hat{s})=1+\frac{\alpha_{\mathrm{s}}(\mu)}{\pi}\left(\frac{164}{9}+\frac{23}{6} \ln \frac{\mu^{2}}{\hat{s}}\right) .
\end{gathered}
$$

$\hat{\sigma}_{g g \rightarrow H Z}^{\mathrm{LO}}$ is again the total inclusive partonic LO cross section, while the "reducible" contribution $\hat{\sigma}_{\text {(virt,red) }}$ is due to Feynman diagrams that involve two quark triangles (see ref. [19] for details). It was calculated in the heavy-top limit in ref. [19]; we use the resulting compact analytic expression in our numerical analysis. In order to approximate the $m_{t}$ dependence, we rescale it by $\hat{\sigma}_{g g \rightarrow H Z}^{\mathrm{LO}} / \hat{\sigma}_{\infty}^{\mathrm{LO}}$, where the LO cross section in the heavy-top limit is given by [19]

$$
\lim _{m_{t} \rightarrow \infty} \hat{\sigma}_{g g \rightarrow H Z}^{\mathrm{LO}}\left(\hat{s}, M_{H}, M_{Z}\right) \equiv \hat{\sigma}_{\infty}^{\mathrm{LO}}\left(\hat{s}, M_{H}, M_{Z}\right)=\kappa \cdot \frac{\lambda^{3 / 2}\left(\hat{s}, M_{H}^{2}, M_{Z}^{2}\right)}{M_{Z}^{4} \hat{s}^{2}},
$$

with

$$
\lambda(x, y, z)=x^{2}+y^{2}+x^{2}-2 x y-2 y z-2 z x
$$

The factor

$$
\kappa=\frac{1}{\pi}\left(\frac{\alpha_{\mathrm{s}} \alpha}{64 c_{\mathrm{W}}^{2} s_{\mathrm{W}}^{2}}\right)^{2}
$$

collects numerical constants that are not directly relevant for the discussion in this section.

Up to $\mathcal{O}\left(\alpha_{\mathrm{s}}\right)$, the hard coefficient in the $Q$-approach then reads

$$
\mathcal{C}_{Q}=K\left(Q^{2}\right)+U\left(Q^{2}\right)+\hat{\sigma}_{(\text {virt,red })} /\left.\hat{\sigma}_{\infty}^{\mathrm{LO}}\right|_{\hat{s}=Q^{2}}
$$

with the universal contribution $U$ defined in eq. (2.12). ${ }^{3}$

\subsection{The absolute threshold limit: $M$-approach}

The $M$-approach requires the Mellin transform of the LO total inclusive cross section $\hat{\sigma}_{g g \rightarrow H Z}^{\mathrm{LO}}$ w.r.t. the variable $\hat{\tau}_{M}=M^{2} / \hat{s}$, which is non-trivial to obtain due to the complicated dependence on $M=M_{H}+M_{Z}$. Since the $M$-approach is concerned with the

\footnotetext{
${ }^{3}$ We note in passing that, apart from the reducible contribution $\sigma_{\text {(virt,red) }}$, the NLO hard coefficient coincides with the one for pseudo-scalar Higgs production in gluon fusion, see ref. [60]. This is expected since it was observed that the corresponding amplitudes for the two processes are the same in the heavy-top limit [19].
} 
logarithms close to the $Z H$ threshold where $\hat{s}<4 m_{t}^{2}$, it is justified to apply the heavy-top limit though, given in eq. (3.5).

Note, however, that $\hat{\sigma}_{\infty}^{\mathrm{LO}}$ diverges as $\hat{s} \rightarrow \infty$. The Mellin integral w.r.t. $\hat{\tau}_{M}=M^{2} / \hat{s}$ therefore does not exist. This is not surprising, since the integration region of the Mellin transform extends to values of $\hat{s}=M^{2} / \hat{\tau}_{M}$ where the heavy-top limit does not hold. In its validity range, however, $\hat{\sigma}_{\infty}^{\mathrm{LO}}$ can safely be approximated by its threshold expansion:

$$
\begin{gathered}
\hat{\sigma}_{\infty}^{\mathrm{LO}}\left(\hat{s}, M_{H}, M_{Z}\right) \stackrel{\hat{s} \rightarrow M^{2}}{\rightarrow} \hat{\sigma}_{\infty, \text { thr. }}^{\mathrm{LO}}\left(\hat{s}, M_{H}, M_{Z}\right)=\frac{8 \kappa}{r_{M} M_{Z}^{2}}\left(r_{M}-1\right)^{3 / 2}\left(1-\hat{\tau}_{M}\right)^{3 / 2}, \\
\text { where } r_{M} \equiv 1+M_{H} / M_{Z}=M / M_{Z},
\end{gathered}
$$

and higher terms in $\left(1-\hat{\tau}_{M}\right)$ have been dropped. Note that the LO cross section tends to zero quite fast at threshold, with one power of $\left(1-\hat{\tau}_{M}\right)^{1 / 2}$ due to the phase space, and two additional powers arising from the squared amplitude. The reason for this behaviour is that at threshold, both the Higgs and the $Z$ are at rest and therefore the $Z H$ system has orbital angular momentum zero. The total angular momentum of the final state is thus given by the spin of the $Z$ boson, $j=1$. Such a configuration of final and initial states is forbidden by the Landau-Yang theorem, and therefore the amplitude must vanish at threshold.

The expression in eq. (3.9) can easily be transformed into Mellin space using

$$
\int_{0}^{1} \mathrm{~d} \hat{\tau}_{M} \hat{\tau}_{M}^{N-1}\left(1-\hat{\tau}_{M}\right)^{3 / 2}=\frac{3 \sqrt{\pi}}{4} \frac{\Gamma(N)}{\Gamma(N+5 / 2)},
$$

with Euler's $\Gamma$-function obeying $z \Gamma(z)=\Gamma(z+1)$ and $\Gamma(1)=1$.

For the determination of the hard matching coefficient $\mathcal{C}_{M}$, we also require the threshold limit (defined in Mellin space) of the NLO virtual corrections. In the $M$-approach, the latter are directly given by eq. (3.4). Having already dealt with $\hat{\sigma}_{g g \rightarrow H Z}^{\mathrm{LO}}$ (the term $\ln \left(\mu^{2} / \hat{s}\right)$ in $K(\hat{s})$ of eq. (3.4) can simply be replaced by $\ln \left(\mu^{2} / M^{2}\right)$ in the threshold limit), what remains is to evaluate the Mellin transform of the reducible terms. Following the same line of argument as for $\hat{\sigma}_{g g \rightarrow H Z}^{\mathrm{LO}}$, we take the threshold limit of the heavy-top limit expression given in ref. [19]:

$$
\begin{aligned}
\hat{\sigma}_{\text {(virt,red) }} & \stackrel{\hat{\tau}_{M} \rightarrow 1}{\rightarrow} \hat{\sigma}_{(\text {virt,red),thr. }} \\
& =-\frac{2}{9} \frac{\alpha_{\mathrm{s}}}{\pi} \hat{\sigma}_{\infty, \mathrm{thr}}^{\mathrm{LO}}\left(\hat{s}, M_{H}, M_{Z}\right)\left[1+r_{M}+\frac{2+r_{M}}{2 r_{M}} \ln \left(r_{M}-1\right)\right] .
\end{aligned}
$$

Note that the threshold behavior of the virtual corrections is the same as for the LO cross section, given by $\hat{\sigma}_{\infty, \text { thr }}^{\mathrm{LO}}$ defined in eq. (3.9). Correspondingly, the Mellin transform can be obtained again using eq. (3.10).

The hard coefficient in the $M$-approach therefore follows as

$$
\mathcal{C}_{M}=K\left(M^{2}\right)+U\left(M^{2}\right)+\hat{\sigma}_{(\text {virt,red),thr. }} / \hat{\sigma}_{\infty, \text { thr. }}^{\mathrm{LO}},
$$

with the functions $K$ and $U$ defined in eqs. (3.4) and (2.12), respectively. Note that the $\hat{\tau}_{M^{-}}$ dependence cancels in the last term on the r.h.s. of eq. (3.12). The final resummed hadronic cross section in the $M$-approach is rescaled by $\sigma_{g g \rightarrow H Z}^{\mathrm{LO}} / \sigma_{\infty, \mathrm{Lhr}}^{\mathrm{LO}}$, in a similar manner to the NLO calculation [19]. 


\section{Results for the resummed cross section}

In this section, we are going to study the numerical impact of the resummed terms on the inclusive total cross section at NLO+NLL, i.e., in eq. (2.13), we set "(f.o.)" $=$ NLO, and "(l.a.)" =NLL. Particular focus will be put on the theoretical uncertainty which is estimated in the usual way by varying the renormalization and the factorization scales around a default value $\mu_{0}$. Furthermore, NNLL effects will be discussed at the end of this section.

At fixed order, $\mu_{0}$ is typically chosen as the invariant mass of the $Z H$ system (see, e.g., ref. [13]), since this is the characteristic scale of the partonic process. In the $Q$ approach, we are going to adopt this choice, which allows us to directly compare to previous predictions of the total cross section. For the $M$-approach, however, the invariant mass is integrated over before the resummation is performed; this scale is therefore unaccessible as central choice for $\mu_{\mathrm{F}}$ or $\mu_{\mathrm{R}}$. In fact, since this approach emphasizes the absolute threshold $M=M_{Z}+M_{H}$, it is this scale that should be considered as default in the $M$-approach.

The other numerical parameters entering the total cross section are chosen as in ref. [3]. In particular, we use $m_{t}=172.5 \mathrm{GeV}$ for the on-shell top mass, and $m_{b}=4.75 \mathrm{GeV}$ for the on-shell bottom mass. Concerning the PDF set, it is not obvious a priori which order is appropriate for the $g g \rightarrow H Z$ process. From a renormalization group and DGLAP point of view, the $g g \rightarrow H Z$ subprocess adds incoherently to the Drell-Yan like terms, so that its $\alpha_{\mathrm{s}}^{2}$ contribution could be considered as LO. The numerical results of ref. [19] were produced by adopting this point of view. On the other hand, the lowest order contribution to $g g \rightarrow H Z$ is $\mathcal{O}\left(\alpha_{\mathrm{s}}^{2}\right)$ which is the same as for the NNLO prediction of the total inclusive Higgs Strahlung process; one could therefore consider it as NNLO. This is the viewpoint taken in ref. [3], and it will also be our default choice. We therefore evaluate all hadronic cross sections using NNLO PDF sets; specifically, we use MSTW2008NNLO. ${ }^{4}$

Figure 2 shows the NLO+NLL result for $8 \mathrm{TeV}$ collisions for the gluon-initiated total inclusive cross section upon simultaneous variation of the unphysical scales, $\mu \equiv \mu_{\mathrm{F}}=\mu_{\mathrm{R}}$, together with the fixed order prediction at LO and NLO, for (a) the $Q$-approach and (b) the $M$-approach. As explained above, all curves have been evaluated with NNLO PDFs. As expected, resummation significantly decreases the scale dependence in both approaches. In accordance with the supposition that the "natural" scale for the partonic process is $Q^{2}=\left(p_{H}+p_{Z}\right)^{2}$, the absolute as well as the relative scale uncertainty is considerably lower for the $Q$-approach. This is mostly due to the fact that the fixed-order results exhibit a much stronger scale dependence upon variation of $\mu$ relative to the fixed scale $M$. For example, while the NLO (LO) cross section varies by $\pm 39 \%$ ( $\pm 65 \%)$ when $\mu$ is changed by a factor of five around the $Z H$ invariant mass, this variation is $\pm 66 \%( \pm 70 \%)$ when the scale is varied by the same factor around the absolute threshold $M=M_{H}+M_{Z}$. The resummed cross section, on the other hand, varies only by $\pm 12 \%$ in the $Q$-approach with $\mu_{0}^{2}=Q^{2}$, and by $\pm 29 \%$ in the $M$-approach with $\mu_{0}=M$. For comparison, figure 2 also shows the NLO+NLL result obtained through the $Q$-approach, but adopting $\mu_{0}=M$ as the central resummation scale. The scale variation is larger $( \pm 43 \%)$ than in the $M$-approach

\footnotetext{
${ }^{4}$ Note that from this perspective, the NLO and NLO+NLL prediction for $g g \rightarrow H Z$ should be evaluated using even higher order PDF sets, which are, however, unavailable to date.
} 


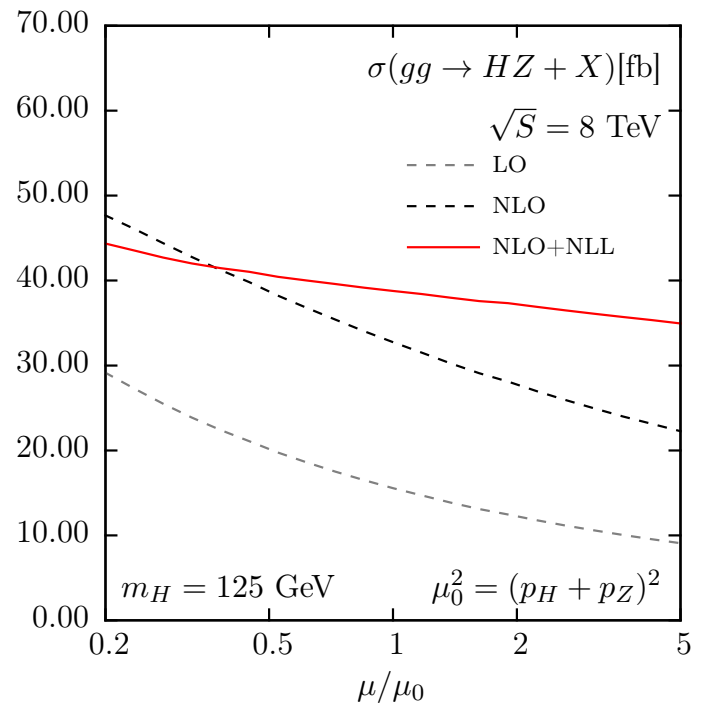

(a)

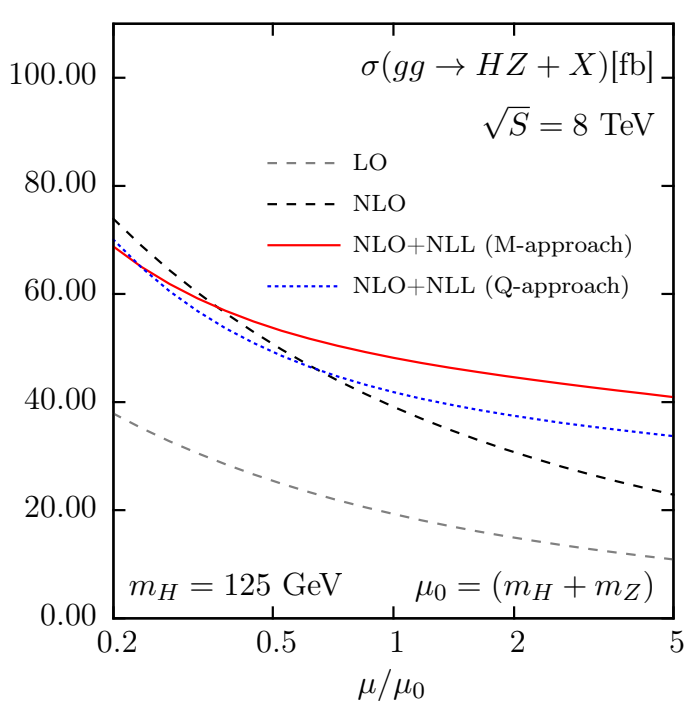

(b)

Figure 2. Total inclusive cross section at $\sqrt{s}=8 \mathrm{TeV}$ due to gluon-induced $Z H$ production at LO (lower dashed; gray), NLO (upper dashed; black), and NLO+NLL (solid; red). (a) $Q$-approach with central scale choice $\mu_{0}^{2}=Q^{2}$, (b) $M$-approach with central scale choice $\mu_{0}^{2}=M^{2}$. Also shown in (b) is the NLO+NLL result from the $Q$-approach for the central scale choice $\mu_{0}^{2}=M^{2}$ (dotted; blue).

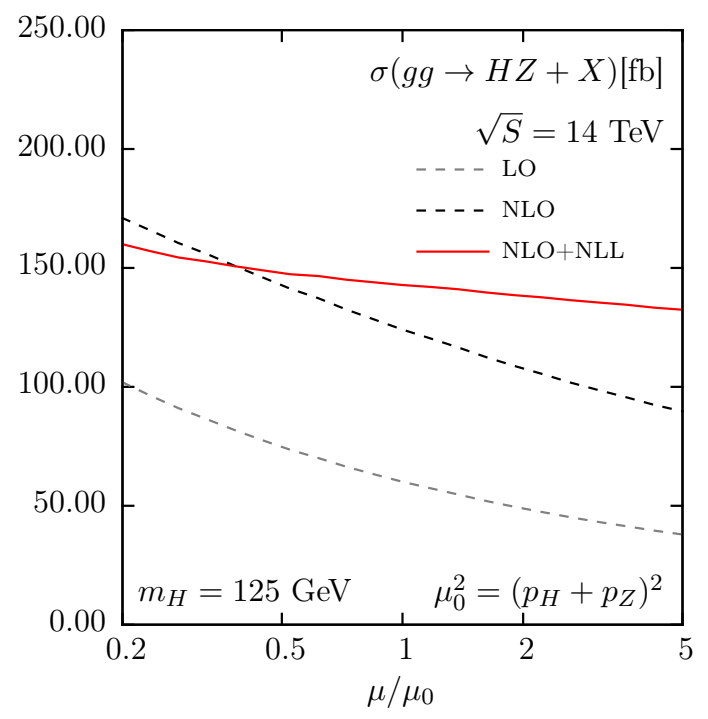

(a)

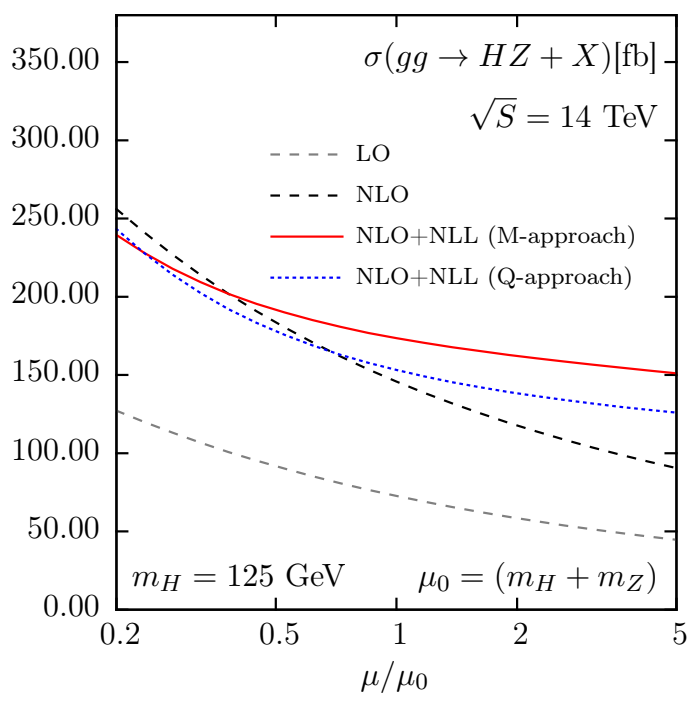

(b)

Figure 3. Same as figure 2, but for $\sqrt{s}=14 \mathrm{TeV}$.

with this scale choice. This is not unexpected, since when $\mu_{0}=M$ is chosen, the decisive logarithms of the ratio $W^{2} / \mu^{2}$ (cf. eq. (2.6)) can get significantly larger for $W^{2}=Q^{2}$ than for $W^{2}=M^{2}$. Correspondingly, as the absolute threshold region gets emphasized relative to configurations with larger invariant mass by the choice $\mu_{0}=M_{H}+M_{Z}$, the effect of resummation in the $M$-approach is more enhanced than in the $Q$-approach. 
As shown in figure 3, apart from an overall increase of the cross section, the behavior of the curves for $p p$ collisions at $\sqrt{s}=14 \mathrm{TeV}$ is almost identical to the case of $8 \mathrm{TeV}$ described above.

Due to these considerations, and in view of the fact that the more suitable scale for the process is considered to be the invariant mass of the $Z H$ system, it is justified to base our final numerical predictions on the results obtained through the $Q$-approach. The comparison with the $M$-approach results is reassuring since the two results are quite compatible within the theoretical uncertainty, even though the central value for the $M$ approach is about $15 \%$ larger than for the $Q$-approach at $\mu_{0}=M_{H}+M_{Z}$ and $\sqrt{s}=8 \mathrm{TeV}$.

Note also that for the central scale choice, we find an increase of the total cross section at $\sqrt{s}=8 \mathrm{TeV}$ of about $18 \%$ relative to the NLO result for the $Q$-approach $(23 \%$ for the $M$-approach), which might be a welcome feature for studies along the lines of refs. [18, 61]. For $\sqrt{s}=14 \mathrm{TeV}$ the corresponding increase of the cross section amounts to $15 \%$.

Before we present our updated prediction for the total inclusive $Z H$ cross section, let us discuss a further addition to the $g g \rightarrow H Z$ subprocess which will, not least, allow us to validate our estimate of the theoretical uncertainty due to missing higher order terms, which will be adopted in section 5 . Since the final state in the $g g \rightarrow H Z$ process is a colour singlet, the radiative factors resumming large logarithmic contributions are process independent and consist only of $\Delta_{g}$ factors, cf. eq. (2.5). Therefore we can easily extend our analysis to include the NNLL terms in the radiative factor $\Delta_{g}$, see eq. (2.6). Note that we will neglect the effect of the NNLO-term of the hard coefficient $\mathcal{C}_{W}$. The calculation of this term will be only possible when the NNLO result for the $g g \rightarrow H Z$ cross section is known. ${ }^{5}$ Therefore we do not consider our predictions including the NNLL radiative factor as final. Instead we use them to validate the stability of the NLO+NLL result.

Our NNLL resummed results are matched to the NLO cross sections. Alternatively, we could have matched the NNLL result to an approximation of the NNLO cross section, consisting of the NLO result and soft terms of NNLO order. However, in this way no essential new information is generated since the soft terms are obtained from an expansion of the resummed result up to NNLO. The difference between the NNLL result matched to NLO and the same result matched to the approximated NNLO is of $\mathcal{O}(1 / N)$ and as such beyond the accuracy of resummation methods which do not control these power-suppressed terms.

Figure 4 compares the NLO+NLL to the NLO+NNLL result in the $Q$-approach. While the upper plot shows the absolute values, in the lower plot the curves encode the relative deviation from what we will use as our central prediction for $\sigma_{g g \rightarrow H Z}$ in the next section, namely the NLO+NLL cross section evaluated at the central value of the renormalization and the factorization scale, $\mu^{2} \equiv \mu_{\mathrm{R}}^{2}=\mu_{\mathrm{F}}^{2}=Q^{2} \equiv\left(p_{H}+p_{Z}\right)^{2}$. We observe that the scale dependence of the NLO+NNLL is slightly smaller than for NLO+NLL, as it is expected due to the higher perturbative orders that are taken into account. However, the NLO+NNLL should only be considered as an indicator of the size of possible higher order effects, since sub-leading terms in $1 / N$ as well as hard contributions at order $\alpha_{s}^{3}$ may still give sizable

\footnotetext{
${ }^{5}$ Note, however, that the hard coefficient at NNLO is given by the corresponding coefficient for the production of a pseudo-scalar particle [60], plus higher order corrections to the reducible terms $\propto \sigma_{(\text {virt,red) }}$, cf. footnote 3 .
} 


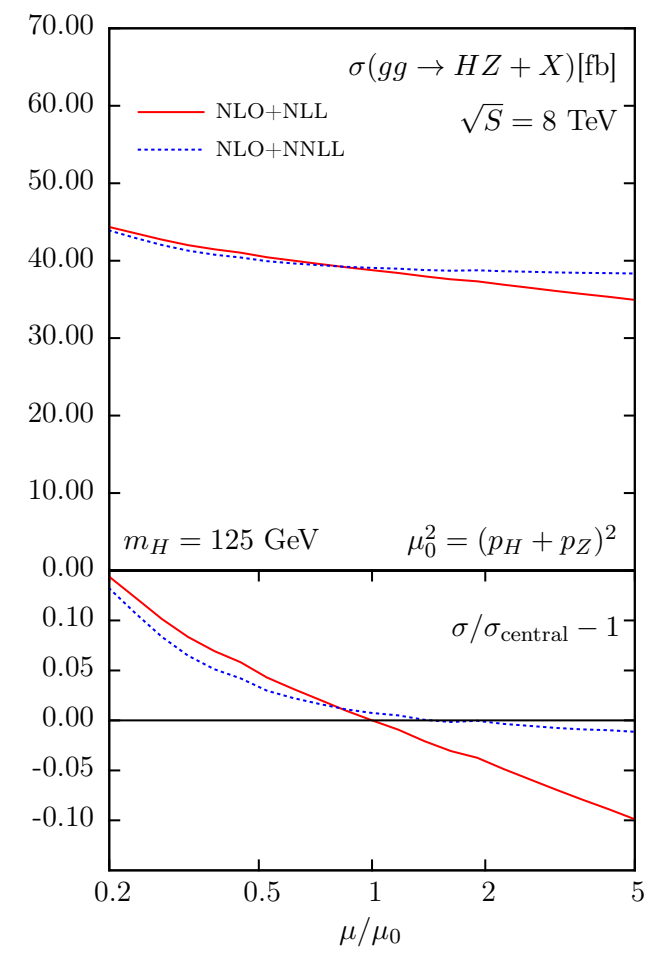

(a)

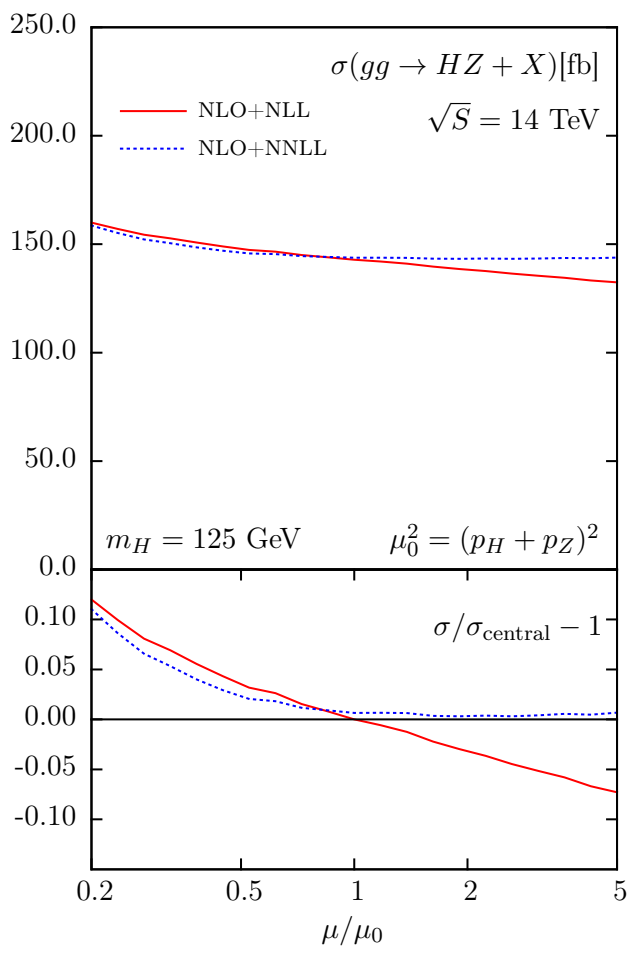

(b)

Figure 4. Total inclusive cross section at (a) $\sqrt{s}=8 \mathrm{TeV}$ and (b) $\sqrt{s}=14 \mathrm{TeV}$ due to gluoninduced $Z H$ production at NLO+NLL (solid; red) and NLO+NNLL (dotted; blue) in the $Q$-approach. Upper plot: absolute values; lower plot: relative deviation to $\sigma_{\text {central }} \equiv \sigma_{g g \rightarrow H Z}\left(\mu^{2}=Q^{2}\right)$, where $Q$ is the invariant mass of the $Z H$ system, and $\mu=\mu_{\mathrm{F}}=\mu_{\mathrm{R}}$ is the renormalization/factorization scale.

contributions. Therefore, we base our theoretical prediction for the total cross section purely on the NLO+NLL result which leads to a slightly more conservative error estimate. ${ }^{6}$

\section{The total inclusive cross section for $Z H$ production}

In this section, we are going to present updated numbers for the total inclusive cross section of associated $Z H$ production at the LHC which, in addition to all previously known effects, takes into account the results of this paper for the resummed $g g \rightarrow H Z$ contributions. We derive the updated prediction by evaluating the non- $g g \rightarrow H Z$ contributions of $Z H$ production and their scale uncertainties using vh@nnlo [18, 40], and adding our new results for the $g g \rightarrow H Z$ component.

The latter are evaluated along the lines of ref. [3], where for the central value, the renormalization and factorization scale is set to the invariant mass of the $Z H$ system, $\mu_{\mathrm{R}}=$ $\mu_{\mathrm{F}}=Q$, while the theoretical error is determined by varying these two scales simultaneously within the interval $[1 / 3,3] Q$. The results for four different energies and three different values

\footnotetext{
${ }^{6}$ Note that any reasonable error band derived from the NLO+NLL result by varying the scale $\mu$ (e.g. within the interval $\left.[1 / 3,3] \mu_{0}\right)$ would cover the analogous band derived from the NLO+NNLL result. This behavior provides quite some confidence in the perturbative stability of the NLO+NLL result.
} 


\begin{tabular}{|c|c|c|c|c|c|}
\hline$\sqrt{s}$ & $M_{H}$ & \multicolumn{4}{|c|}{$\sigma_{g g \rightarrow H Z}$} \\
\hline$[\mathrm{TeV}]$ & {$[\mathrm{GeV}]$} & \multicolumn{2}{|c|}{$\mathrm{NLO}$} & \multicolumn{2}{|c|}{$\mathrm{NLO}+\mathrm{NLL}$} \\
\cline { 3 - 6 } & & {$[\mathrm{pb}]$} & {$[\%]$} & {$[\mathrm{pb}]$} & {$[\%]$} \\
\hline 8.00 & 125.0 & 0.0328 & ${ }_{-23}^{+30}$ & 0.0389 & ${ }_{-6.5}^{+8.1}$ \\
8.00 & 125.5 & 0.0325 & ${ }_{-23}^{+30}$ & 0.0386 & ${ }_{-6.5}^{+8.1}$ \\
8.00 & 126.0 & 0.0324 & ${ }_{-23}^{+30}$ & 0.0384 & ${ }_{-6.6}^{+8.2}$ \\
13.0 & 125.0 & 0.1057 & ${ }_{-21}^{+26}$ & 0.1220 & ${ }_{-5.2}^{+7.0}$ \\
13.0 & 125.5 & 0.1049 & ${ }_{-20}^{+26}$ & 0.1211 & ${ }_{-5.2}^{+7.3}$ \\
13.0 & 126.0 & 0.1044 & ${ }_{-21}^{+26}$ & 0.1205 & ${ }_{-5.5}^{+6.7}$ \\
13.5 & 125.0 & 0.1149 & ${ }_{-20}^{+26}$ & 0.1325 & ${ }_{-5.2}^{+7.1}$ \\
13.5 & 125.5 & 0.1141 & ${ }_{-20}^{+26}$ & 0.1316 & ${ }_{-5.0}^{+7.2}$ \\
13.5 & 126.0 & 0.1135 & ${ }_{-20}^{+26}$ & 0.1308 & ${ }_{-5.0}^{+7.1}$ \\
14.0 & 125.0 & 0.1243 & ${ }_{-20}^{+26}$ & 0.1431 & ${ }_{-4.9}^{+7.1}$ \\
14.0 & 125.5 & 0.1237 & ${ }_{-20}^{+26}$ & 0.1424 & ${ }_{-5.0}^{+7.3}$ \\
14.0 & 126.0 & 0.1228 & ${ }_{-20}^{+26}$ & 0.1414 & ${ }_{-4.9}^{+7.3}$ \\
\hline
\end{tabular}

Table 1. The $g g \rightarrow H Z$ component to the total inclusive $Z H$ production cross section for various center-of-mass energies $\sqrt{s}$ and Higgs masses $M_{H}$. The third/fourth column displays the NLO result, while the fifth/sixth column shows the NLO+NLL prediction (based on the $Q$-approach) calculated in this paper. The uncertainties (column 4 and 6) are due to scale variation.

of the Higgs mass are shown in table 1. For comparison, we have also included the numbers of the NLO prediction. The most notable effect of the NLO+NLL result for $g g \rightarrow H Z$ is the reduction of the theoretical error due to scale variation by a factor of three to four. Also the central value of the total inclusive rate increases by about $18 \%$ for $\sqrt{s}=8 \mathrm{TeV}$, and by about $15 \%$ at 13 and $14 \mathrm{TeV}$.

Let us now see how these effects translate to the total inclusive cross section for the process $p p \rightarrow Z H$. In order to obtain the central value of the total cross section, we add the central values of the $g g \rightarrow H Z$ and non- $g g \rightarrow H Z$ components. The scale error of the latter is evaluated along the lines of ref. [3] by varying $\mu_{\mathrm{F}}$ and $\mu_{\mathrm{R}}$ independently within the interval $[1 / 3,3] Q$, and added linearly to the scale errors of the $g g \rightarrow H Z$ contribution. Our final prediction is displayed in table 2 for the same energies and Higgs masses as before. For comparison, we have again included the numbers based on the NLO prediction of the $g g \rightarrow H Z$ process which correspond to the current recommended numbers of the LHCHXSWG $[3-5,62,63] .^{7}$ The effect of the reduction of the scale variation in the $g g \rightarrow H Z$ subprocess on the total cross section is still significant, amounting to more than a factor

\footnotetext{
${ }^{7}$ The current numbers of the LHC-HXSWG at $\sqrt{s}=8 \mathrm{TeV}$ have a symmetrized error, thus they slightly differ from the ones listed in table 2 .
} 


\begin{tabular}{|c|c|c|c|c|c|}
\hline$\sqrt{s}$ & $M_{H}$ & \multicolumn{4}{|c|}{$\sigma_{p p \rightarrow H Z}$} \\
\hline$[\mathrm{TeV}]$ & {$[\mathrm{GeV}]$} & \multicolumn{2}{|c|}{ incl. } & \multicolumn{2}{|c|}{ incl. } \\
& & $g g @$ @LO & \multicolumn{2}{|c|}{$g g @$ NLO+NLL } \\
\cline { 3 - 6 } & & {$[\mathrm{pb}]$} & {$[\%]$} & {$[\mathrm{pb}]$} & {$[\%]$} \\
\hline 8.00 & 125.0 & 0.4157 & ${ }_{-2.8}^{+3.1}$ & 0.4217 & ${ }_{-1.5}^{+1.5}$ \\
8.00 & 125.5 & 0.4104 & ${ }_{-2.8}^{+3.1}$ & 0.4165 & ${ }_{-1.5}^{+1.5}$ \\
8.00 & 126.0 & 0.4054 & ${ }_{-2.8}^{+3.2}$ & 0.4114 & ${ }_{-1.6}^{+1.5}$ \\
13.0 & 125.0 & 0.8696 & ${ }_{-3.8}^{+3.8}$ & 0.8859 & ${ }_{-2.0}^{+1.6}$ \\
13.0 & 125.5 & 0.8594 & ${ }_{-3.8}^{+3.8}$ & 0.8757 & ${ }_{-1.9}^{+1.7}$ \\
13.0 & 126.0 & 0.8501 & ${ }_{-3.9}^{+3.8}$ & 0.8663 & ${ }_{-2.1}^{+1.6}$ \\
13.5 & 125.0 & 0.9190 & ${ }_{-3.8}^{+3.9}$ & 0.9366 & ${ }_{-2.0}^{+1.6}$ \\
13.5 & 125.5 & 0.9085 & ${ }_{-3.8}^{+3.8}$ & 0.9259 & ${ }_{-2.0}^{+1.6}$ \\
13.5 & 126.0 & 0.8988 & ${ }_{-3.9}^{+3.8}$ & 0.9162 & ${ }_{-2.0}^{+1.5}$ \\
14.0 & 125.0 & 0.9690 & ${ }_{-3.9}^{+4.0}$ & 0.9878 & ${ }_{-2.0}^{+1.7}$ \\
14.0 & 125.5 & 0.9574 & ${ }_{-3.9}^{+4.0}$ & 0.9761 & ${ }_{-2.0}^{+1.7}$ \\
14.0 & 126.0 & 0.9465 & ${ }_{-3.9}^{+4.1}$ & 0.9652 & ${ }_{-2.0}^{+1.7}$ \\
\hline
\end{tabular}

Table 2. Total inclusive cross section for the process $p p \rightarrow Z H$ for various center-of-mass energies $\sqrt{s}$ and Higgs masses $M_{H}$. The third/fourth column includes the $g g \rightarrow H Z$ subprocess through NLO, while the fifth/sixth column includes the NLO+NLL prediction (based on the $Q$-approach) for the $g g \rightarrow H Z$ subprocesses as calculated in this paper. The uncertainties (column 4 and 6 ) are due to scale variation. $\mathrm{PDF}+\alpha_{s}$ uncertainties are at the few-percent level and are not listed here; they can be taken over from the LHC-HXSWG recommendations [62, 63].

of two in many cases. This reflects the dominance of the $g g \rightarrow H Z$ error in the total uncertainty of this process, which can be inferred also by comparing the scale error of the $Z H$ cross section to the one for $W H$ production. The central value of the total inclusive rate increases by about $1.5 \%$ for $\sqrt{s}=8 \mathrm{TeV}$, and by about $2 \%$ at 13 and $14 \mathrm{TeV}$.

\section{Conclusions}

We have presented the threshold resummed cross section for gluon-induced $Z H$ production through NLL, matched to the NLO result. Two approaches were pursued: in the $Q$-approach, logarithms relative to the threshold of producing a $Z H$ pair of invariant mass $Q$ were resummed, while in the $M$-approach, the resummation was performed relative to the absolute threshold of the $Z H$ system, $M=M_{H}+M_{Z}$. It was argued that, due to the possibility of a more appropriate choice for the renormalization and the factorization scale, the $Q$-approach leads to perturbatively more stable and thus more precise predictions for the $g g \rightarrow H Z$ cross section. In order to underline the reliability of our prediction, we 
considered the universal terms arising at NNLL and found complete compatibility with the $\mathrm{NLO}+\mathrm{NLL}$ result.

The NLO+NLL result was found to exhibit a strongly reduced scale variation relative to the NLO result, while the central value was increased. This motivates a re-evaluation of the total inclusive $Z H$ cross section, including the previously known NNLO corrections to the "Drell-Yan-like" contributions [13] as well as top-loop induced effects at $\alpha_{s}^{2}$ [14]. The central value of the new prediction for $\sigma(p p \rightarrow H Z)$ is slightly larger than the value currently suggested by the LHC-HXSWG, and the theoretical error due to scale variation is reduced by roughly a factor of two.

Acknowledgments. We thank Stefan Liebler for assistance with the $g g \rightarrow H Z$ subroutines of vh@nnlo, and Fabio Maltoni for useful discussions. This research was supported by the Munich Institute for Astro- and Particle Physics (MIAPP) of the DFG cluster of excellence "Origin and Structure of the Universe", and by the DFG contract HA 2990/5-1. The support by Polish National Science Centre grant, project number DEC2011/01/B/ST2/03643, is gratefully acknowledged.

Open Access. This article is distributed under the terms of the Creative Commons Attribution License (CC-BY 4.0), which permits any use, distribution and reproduction in any medium, provided the original author(s) and source are credited.

\section{References}

[1] ATLAS collaboration, Observation of a new particle in the search for the standard model Higgs boson with the ATLAS detector at the LHC, Phys. Lett. B 716 (2012) 1 [arXiv: 1207.7214] [INSPIRE].

[2] CMS collaboration, Observation of a new boson at a mass of $125 \mathrm{GeV}$ with the CMS experiment at the LHC, Phys. Lett. B 716 (2012) 30 [arXiv:1207.7235] [INSPIRE].

[3] LHC Higgs Cross Section Working Group collaboration, S. Heinemeyer et al., Handbook of LHC Higgs cross sections: 3. Higgs properties, arXiv:1307.1347 [INSPIRE].

[4] S. Dittmaier et al., Handbook of LHC Higgs cross sections: 2. Differential distributions, arXiv: 1201.3084 [INSPIRE].

[5] LHC Higgs Cross Section Working Group collaboration, S. Dittmaier et al., Handbook of LHC Higgs cross sections: 1. Inclusive observables, arXiv:1101.0593 [INSPIRE].

[6] J.M. Butterworth, A.R. Davison, M. Rubin and G.P. Salam, Jet substructure as a new Higgs search channel at the LHC, Phys. Rev. Lett. 100 (2008) 242001 [arXiv:0802.2470] [INSPIRE].

[7] T. Han and S. Willenbrock, $Q C D$ correction to the $p p \rightarrow W H$ and $Z H$ total cross-sections, Phys. Lett. B 273 (1991) 167 [INSPIRE].

[8] H. Baer, B. Bailey and J.F. Owens, $O\left(\alpha_{s}\right)$ Monte Carlo approach to $W+$ Higgs associated production at hadron supercolliders, Phys. Rev. D 47 (1993) 2730 [INSPIRE].

[9] J. Ohnemus and W.J. Stirling, Order $\alpha_{s}$ corrections to the differential cross-section for the WH intermediate mass Higgs signal, Phys. Rev. D 47 (1993) 2722 [INSPIRE]. 
[10] S. Mrenna and C.P. Yuan, Effects of QCD resummation on $W+h$ and $t \bar{b}$ production at the Tevatron, Phys. Lett. B 416 (1998) 200 [hep-ph/9703224] [INSPIRE].

[11] M. Spira, QCD effects in Higgs physics, Fortsch. Phys. 46 (1998) 203 [hep-ph/9705337] [INSPIRE].

[12] A. Djouadi and M. Spira, SUSY-QCD corrections to Higgs boson production at hadron colliders, Phys. Rev. D 62 (2000) 014004 [hep-ph/9912476] [INSPIRE].

[13] O. Brein, A. Djouadi and R. Harlander, NNLO QCD corrections to the Higgs-strahlung processes at hadron colliders, Phys. Lett. B 579 (2004) 149 [hep-ph/0307206] [INSPIRE].

[14] O. Brein, R. Harlander, M. Wiesemann and T. Zirke, Top-quark mediated effects in hadronic Higgs-Strahlung, Eur. Phys. J. C 72 (2012) 1868 [arXiv:1111.0761] [INSPIRE].

[15] G. Ferrera, M. Grazzini and F. Tramontano, Associated $W H$ production at hadron colliders: a fully exclusive QCD calculation at NNLO, Phys. Rev. Lett. 107 (2011) 152003 [arXiv: 1107.1164] [INSPIRE].

[16] G. Ferrera, M. Grazzini and F. Tramontano, Associated $Z H$ production at hadron colliders: the fully differential NNLO QCD calculation, arXiv:1407.4747 [INSPIRE].

[17] B.A. Kniehl and C.P. Palisoc, Associated production of $Z$ and neutral Higgs bosons at the CERN Large Hadron Collider, Phys. Rev. D 85 (2012) 075027 [arXiv:1112.1575] [InSPIRE].

[18] R.V. Harlander, S. Liebler and T. Zirke, Higgs Strahlung at the Large Hadron Collider in the 2-Higgs-doublet model, JHEP 02 (2014) 023 [arXiv: 1307.8122] [INSPIRE].

[19] L. Altenkamp, S. Dittmaier, R.V. Harlander, H. Rzehak and T.J.E. Zirke, Gluon-induced Higgs-strahlung at next-to-leading order QCD, JHEP 02 (2013) 078 [arXiv:1211.5015] [INSPIRE].

[20] A. Djouadi, The anatomy of electro-weak symmetry breaking. I: The Higgs boson in the standard model, Phys. Rept. 457 (2008) 1 [hep-ph/0503172] [INSPIRE].

[21] S. Catani, D. de Florian, M. Grazzini and P. Nason, Soft gluon resummation for Higgs boson production at hadron colliders, JHEP 07 (2003) 028 [hep-ph/0306211] [INSPIRE].

[22] A. Kulesza, G.F. Sterman and W. Vogelsang, Joint resummation for Higgs production, Phys. Rev. D 69 (2004) 014012 [hep-ph/0309264] [INSPIRE].

[23] S. Moch and A. Vogt, Higher-order soft corrections to lepton pair and Higgs boson production, Phys. Lett. B 631 (2005) 48 [hep-ph/0508265] [INSPIRE].

[24] E. Laenen and L. Magnea, Threshold resummation for electroweak annihilation from DIS data, Phys. Lett. B 632 (2006) 270 [hep-ph/0508284] [INSPIRE].

[25] V. Ravindran, On Sudakov and soft resummations in QCD, Nucl. Phys. B 746 (2006) 58 [hep-ph/0512249] [INSPIRE].

[26] V. Ravindran, Higher-order threshold effects to inclusive processes in QCD, Nucl. Phys. B 752 (2006) 173 [hep-ph/0603041] [INSPIRE].

[27] A. Idilbi, X.-d. Ji, J.-P. Ma and F. Yuan, Threshold resummation for Higgs production in effective field theory, Phys. Rev. D 73 (2006) 077501 [hep-ph/0509294] [INSPIRE].

[28] A. Idilbi, X.-d. Ji and F. Yuan, Resummation of threshold logarithms in effective field theory for DIS, Drell-Yan and Higgs production, Nucl. Phys. B 753 (2006) 42 [hep-ph/0605068] [INSPIRE]. 
[29] V. Ahrens, T. Becher, M. Neubert and L.L. Yang, Origin of the large perturbative corrections to Higgs production at hadron colliders, Phys. Rev. D 79 (2009) 033013 [arXiv:0808.3008] [INSPIRE].

[30] V. Ahrens, T. Becher, M. Neubert and L.L. Yang, Renormalization-group improved prediction for Higgs production at hadron colliders, Eur. Phys. J. C 62 (2009) 333 [arXiv: 0809.4283] [INSPIRE].

[31] R.D. Ball, M. Bonvini, S. Forte, S. Marzani and G. Ridolfi, Higgs production in gluon fusion beyond NNLO, Nucl. Phys. B 874 (2013) 746 [arXiv:1303.3590] [InSPIRE].

[32] M. Bonvini and S. Marzani, Resummed Higgs cross section at $N^{3} L L$, JHEP 09 (2014) 007 [arXiv: 1405.3654] [INSPIRE].

[33] D. de Florian, A. Kulesza and W. Vogelsang, Threshold resummation for high-transverse-momentum Higgs production at the LHC, JHEP 02 (2006) 047 [hep-ph/0511205] [INSPIRE].

[34] F.P. Huang, C.S. Li, H.T. Li and J. Wang, Renormalization-group improved predictions for Higgs boson production at large $p_{T}$, arXiv:1406.2591 [INSPIRE].

[35] T. Becher, G. Bell, C. Lorentzen and S. Marti, The transverse-momentum spectrum of Higgs bosons near threshold at NNLO, arXiv:1407.4111 [INSPIRE].

[36] S. Dawson, T. Han, W.K. Lai, A.K. Leibovich and I. Lewis, Resummation effects in vector-boson and Higgs associated production, Phys. Rev. D 86 (2012) 074007 [arXiv: 1207.4207] [INSPIRE].

[37] G.F. Sterman, Summation of large corrections to short distance hadronic cross-sections, Nucl. Phys. B 281 (1987) 310 [INSPIRE].

[38] S. Catani and L. Trentadue, Resummation of the QCD perturbative series for hard processes, Nucl. Phys. B 327 (1989) 323 [inSPIRE].

[39] M. Czakon, A. Mitov and G.F. Sterman, Threshold resummation for top-pair hadroproduction to next-to-next-to-leading log, Phys. Rev. D 80 (2009) 074017 [arXiv:0907.1790] [INSPIRE].

[40] O. Brein, R.V. Harlander and T.J.E. Zirke, VH@NNLO - Higgs Strahlung at hadron colliders, Comput. Phys. Commun. 184 (2013) 998 [arXiv:1210.5347] [INSPIRE].

[41] H. Mantler and M. Wiesemann, Top- and bottom-mass effects in hadronic Higgs production at small transverse momenta through LO+NLL, Eur. Phys. J. C 73 (2013) 2467 [arXiv: 1210.8263] [INSPIRE].

[42] M. Grazzini and H. Sargsyan, Heavy-quark mass effects in Higgs boson production at the LHC, JHEP 09 (2013) 129 [arXiv: 1306.4581] [INSPIRE].

[43] A. Banfi, P.F. Monni and G. Zanderighi, Quark masses in Higgs production with a jet veto, JHEP 01 (2014) 097 [arXiv: 1308.4634] [INSPIRE].

[44] R.V. Harlander, H. Mantler and M. Wiesemann, Transverse momentum resummation for Higgs production via gluon fusion in the MSSM, arXiv:1409.0531 [INSPIRE].

[45] J. Kodaira and L. Trentadue, Summing soft emission in QCD, Phys. Lett. B 112 (1982) 66 [INSPIRE].

[46] S. Catani, B.R. Webber and G. Marchesini, QCD coherent branching and semiinclusive processes at large $x$, Nucl. Phys. B 349 (1991) 635 [INSPIRE]. 
[47] S. Moch, J.A.M. Vermaseren and A. Vogt, Higher-order corrections in threshold resummation, Nucl. Phys. B 726 (2005) 317 [hep-ph/0506288] [INSPIRE].

[48] A. Vogt, Next-to-next-to-leading logarithmic threshold resummation for deep inelastic scattering and the Drell-Yan process, Phys. Lett. B 497 (2001) 228 [hep-ph/0010146] [INSPIRE].

[49] S. Catani, D. de Florian and M. Grazzini, Higgs production in hadron collisions: Soft and virtual QCD corrections at NNLO, JHEP 05 (2001) 025 [hep-ph/0102227] [INSPIRE].

[50] S. Catani and M.H. Seymour, A general algorithm for calculating jet cross-sections in NLO QCD, Nucl. Phys. B 485 (1997) 291 [Erratum ibid. B 510 (1998) 503] [hep-ph/9605323] [INSPIRE].

[51] J. Blümlein and S. Kurth, Harmonic sums and Mellin transforms up to two loop order, Phys. Rev. D 60 (1999) 014018 [hep-ph/9810241] [INSPIRE].

[52] S. Catani, L. Cieri, D. de Florian, G. Ferrera and M. Grazzini, Universality of transverse-momentum resummation and hard factors at the NNLO, Nucl. Phys. B 881 (2014) 414 [arXiv: 1311.1654] [INSPIRE].

[53] S. Catani, M.L. Mangano, P. Nason and L. Trentadue, The resummation of soft gluons in hadronic collisions, Nucl. Phys. B 478 (1996) 273 [hep-ph/9604351] [INSPIRE].

[54] V. Ahrens, A. Ferroglia, M. Neubert, B.D. Pecjak and L.L. Yang, Renormalization-group improved predictions for top-quark pair production at hadron colliders, JHEP 09 (2010) 097 [arXiv: 1003.5827] [INSPIRE].

[55] M. Beneke, P. Falgari, S. Klein and C. Schwinn, Hadronic top-quark pair production with NNLL threshold resummation, Nucl. Phys. B 855 (2012) 695 [arXiv:1109.1536] [InSPIRE].

[56] B.A. Kniehl, Associated production of Higgs and $Z$ bosons from gluon fusion in hadron collisions, Phys. Rev. D 42 (1990) 2253 [InSPIRE].

[57] B.A. Kniehl, Elastic ep scattering and the Weizsacker-Williams approximation, Phys. Lett. B 254 (1991) 267 [INSPIRE].

[58] D.A. Dicus and C. Kao, Higgs boson-Z $Z^{0}$ production from gluon fusion, Phys. Rev. D 38 (1988) 1008 [Erratum ibid. D 42 (1990) 2412] [INSPIRE].

[59] T. Hahn, Automatic loop calculations with FeynArts, FormCalc and LoopTools, Nucl. Phys. Proc. Suppl. 89 (2000) 231 [hep-ph/0005029] [INSPIRE].

[60] D. de Florian and J. Zurita, Soft-gluon resummation for pseudoscalar Higgs boson production at hadron colliders, Phys. Lett. B 659 (2008) 813 [arXiv:0711.1916] [INSPIRE].

[61] C. Englert, M. McCullough and M. Spannowsky, Gluon-initiated associated production boosts Higgs physics, Phys. Rev. D 89 (2014) 013013 [arXiv: 1310.4828] [INSPIRE].

[62] https://twiki.cern.ch/twiki/bin/view/LHCPhysics/CERNYellowReportPageAt8TeV.

[63] https://twiki.cern.ch/twiki/bin/view/LHCPhysics/CERNYellowReportPageAt1314TeV. 